\title{
Two results on compressor surge control with disturbance rejection
}

\author{
Jan Tommy Gravdahl and Olav Egeland \\ Department of Engineering Cybernetics \\ Norwegian University of Science and Technology \\ O.S. Bragstads plass 8, N-7034 Trondheim, Norway \\ Email: \{Tommy.Gravdahl,Olav.Egeland\}@itk.ntnu.no
}

\begin{abstract}
This paper presents two results on design of compressor surge controllers with disturbance rejection capabilities. First, constant disturbances are included in the Greitzer model, and adaptive backstepping is employed to derive a surge control law for a close coupled valve. Global asymptotic stability is proven. Then, time varying disturbances are studied. A controller is derived using passivity, rendering the closed loop system $\mathcal{L}_{2}$-stable.
\end{abstract}

\section{Introduction}

This paper presents results from an investigation on nonlinear compressor control where feedback is used to stabilize the compressor to the left of the surge line. The useful range of operation of turbocompressors is limited, by choking for high mass flows when sonic velocity is reached in some component, and for low mass flows by the onset of two instabilities known as surge and rotating stall. In this paper surge will be in focus. Surge is an axisymmtrical oscillation of the flow through the compressor, and is characterized by a limit oscillation in the compressor map. Surge oscillations are in most applications unwanted, and can in extreme cases even damage the compressor. Surge can also induce vibrations in other components of the compression system, such as e.g. connected piping [3, 8]. It is common to distinguish between at least two different types of surge: 1) Mild/Classic surge and 2) Deep surge. A combination of surge and rotating stall is known as modified surge. The first of these types is a phenomenon with oscillations in both pressure and flow in the compressor system, while in the second type, the oscillations in mass flow have such a large amplitude, that flow reversal occurs in the compression system. Surge can occur in both axial and centrifugal compressors. It was shown by [11] that the surge model of [7], which was derived for an axial compression system and is going to be used in this paper, also is applicable to a centrifugal compression system.

A constant or slow varying disturbance in mass flow can cause the equilibrium of the compression system to be moved into the unstable area of the compressor map. Therefore, the problem of stabilizing the system in this case is a very important one. In addition constant disturbances in pressure will also be studied.

This instability problem has been extensively studied, and industrial solutions based on surge avoidance are well established. These solutions are based on keeping the operating point to the right of the compressor surge line using a surge margin. There is a potential for 1) increasing the efficiency of compressors by allowing for operation closer to the surge line, and 2) increasing the range of mass flows over which the compressor can operate stably. This, raises the need for control techniques, which stabilize the compressor also to the left of the surge line, as disturbances or set point changes may cause crossing of the surge line if the surge margin is small. This increase in efficiency and mass flow range is in particular possible with compressor designs where the design is done with such controllers in mind.

Among several possible actuators for stabilizing compression systems, the use of a close-coupled valve (hereafter named $\mathrm{CCV}$ ) has been claimed to be among the most promising actuators. Other actuators includes the throttle, bleed valves, loudspeakers, recirculation valves, movable walls and so on. The use of a CCV for control of compressor surge was studied in [8], [16], [17], [13] and [5]. Experimental results of compressor surge control using a CCV was reported in [3] and [2]. In [17] and this strategy was compared, using linear theory, to a number of other possible methods of actuation and also sensing. The conclusion was that the most promising method of surge control is to actuate the system with feedback from the mass flow measurement to a CCV or an injector. In line with this conclusion are the recent results on I/O-selection of [19], where controllers were derived based on $\mathcal{H}_{\infty}$ performance specifications.

\section{Modeling}

\subsection{Compression system}

A compression system consisting of a inlet duct, compressor, plenum volume and throttle valve is studied. Such a system was modeled by [7], and the following model will be used in this paper:

$$
\begin{aligned}
\dot{\Phi} & =\frac{1}{l_{c}}\left(\Psi_{c}(\Phi)-\Psi\right) \\
\dot{\Psi} & =\frac{1}{4 B^{2} l_{c}}\left(\Phi-\Phi_{T}(\Psi)\right),
\end{aligned}
$$

where $\Phi$ is the mass flow coefficient (axial velocity divided by compressor speed), $\Psi$ is the non dimensional plenum pressure or pressure coefficient (pressure divided by density and the square of compressor speed), $\Psi_{c}(\Phi)=\psi_{c 0}+H\left(1+\frac{3}{2}\left(\frac{\Phi}{W}-1\right)-\frac{1}{2}\left(\frac{\Phi}{W}-1\right)^{3}\right)$ is the compressor characteristic, where $W, H$ and $\psi_{c 0}$ are constants, $\Phi_{T}(\Psi)=\gamma_{T} \sqrt{\Psi}$ is the throttle mass flow coefficient, where $\gamma_{T}>0$ is the throttle setting. Greitzer's B-parameter is defined in [7] as $B \triangleq \frac{U}{2 a_{s}} \sqrt{\frac{V_{p}}{A_{c} L_{c}}}$ where $U$ is the constant compressor speed, $a_{s}$ is the speed of sound, $V_{p}$ is the plenum volume, $A_{c}$ is the flow area and $L_{c}$ is the length of ducts and compressor. $l_{c}=\frac{L_{c}}{R}$ is a nondimensional length, were $R$ is the mean compressor radius. The time variable $\xi$ used throughout this paper is also nondimensional, and is defined as 
$\xi \triangleq U t / R$, where $t$ is the actual time. The notation $\dot{\Phi}$ is to be understood as the derivative of $\Phi$ wrt $\xi$.

A compressor in series with a CCV will be studied in the following. The compression system is shown in Figure 1. With close-coupled is understood that the dis-

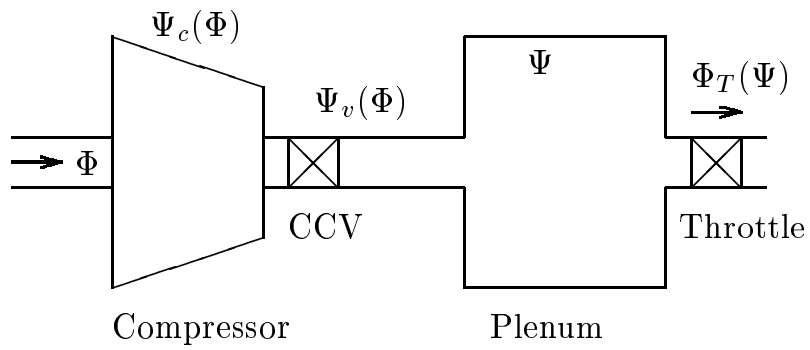

Figure 1: Compression system with $C C V$

tance between the compressor outlet and the valve is so small that no significant mass storage can take place [16]. The assumption of no mass storage between the compressor and the valve allows for the definition of a equivalent compressor. The pressure rise over this equivalent compressor is the sum of the pressure rise over the compressor and the pressure drop over the valve. The pressure drop over the valve will be used as the control, and allow for manipulation of the equivalent compressor characteristic given by

$$
\Psi_{e}(\Phi)=\Psi_{c}(\Phi)-\Psi_{v}(\Phi),
$$

where $\Psi_{c}(\Phi)$ and $\Psi_{v}(\Phi)$ are the compressor pressure rise and valve pressure drop respectively and $\Phi$ is the axial mass flow coefficient. The $\mathrm{CCV}$ has a characteristic given by

$$
\Psi_{v}(\Phi)=\frac{1}{\gamma^{2}} \Phi^{2}
$$

where $\gamma>0$ is proportional to the valve opening. In the model (1), $\Psi_{c}$ is now replaced by $\Psi_{e}$.

The equilibrium $\left(\phi_{0}, \psi_{0}\right)$ of the compression system without the presence of the valve, is located at the intersection of the compressor characteristic $\Psi_{c}(\Phi)$ and the throttle characteristic $\Psi_{T}(\Phi) \triangleq \Phi_{T}^{-1}(\Phi)$. Generally, if the slope $\frac{d \Psi_{c}(\Phi)}{d \Phi}$ of the compressor characteristic is positive at this intersection, the equilibrium is unstable and the compressor will enter surge oscillations. If $\frac{d \Psi_{c}(\Phi)}{d \Phi}<0$ at the intersection, the equilibrium is stable. When introducing the CCV into the system, the equilibrium is shifted to the intersection of the equivalent compressor characteristic $\Psi_{e}(\Phi)$ and the throttle characteristic, and the sign of the slope of $\Psi_{e}(\Phi)$ determines the stability properties of this equilibrium. Thus, by using the pressure drop over the CCV as the control, we are able to manipulate the slope of the equivalent compressor and thereby influence the stability properties of the equilibrium.

To prepare for the analysis of the system, it is desirable to perform a change of coordinates on the system equations so that the origin becomes the equilibrium under study. The new coordinates are defined as

$$
\hat{\psi}=\Psi-\psi_{0} \text { and } \hat{\phi}=\Phi-\phi_{0} \text {. }
$$

It can be shown [4] that the compression system model in these new coordinates is given by

$$
\dot{\hat{\psi}}=\frac{1}{4 B^{2} l_{c}}\left(\hat{\phi}-\hat{\Phi}_{T}(\hat{\psi})\right)
$$

$$
\dot{\hat{\phi}}=\frac{1}{l_{c}}\left(\hat{\Psi}_{c}(\hat{\phi})-u-\hat{\psi}\right),
$$

where

$$
\hat{\Psi}_{c}(\hat{\phi})=-k_{3} \hat{\phi}^{3}-k_{2} \hat{\phi}^{2}-k_{1} \hat{\phi}
$$

and

$$
u=\hat{\Psi}_{v}(\hat{\phi})+\psi_{0}-\Psi_{v}\left(\phi_{0}\right)=\Psi_{v}(\Phi)-\Psi_{v}\left(\phi_{0}\right) .
$$

The constants $k_{1}, k_{2}$ and $k_{3}$ are defined in [4].

\subsection{Disturbances}

As in all physical systems, disturbances will occur in the compression system. In [9] it is stated that this is a topic that need more study, at least in the case of the disturbances initiating stall and surge. Some research have been done in this area. In [12] and [1] circumferential inlet distortion is studied, and in [16] and [10] mass flow and pressure disturbances are investigated.

As in [16] the effect of a pressure disturbance $\hat{\Psi}_{d}(\xi)$, and a flow disturbance $\hat{\Phi}_{d}(\xi)$ will be considered here. The pressure disturbance, which may arise from combustion induced fluctuations when considering the model of a gas turbine, will accelerate the flow. The flow disturbances may arise from processes upstream of the compressor, other compressors in series or air cleaner in the compressor duct, [18]. In the case of the compression system being a aircraft jet engine, large angle of attack or altitude variations may cause flow disturbances, [18].

In the analysis of $[16] \hat{\Phi}_{d}(\xi)$ is set to zero. Disturbances in stall/surge control are also studied in [10]. Here, both types of disturbances, mass flow and pressure, will be considered. The disturbances are time varying, and as in [10] it is assumed that $\hat{\Phi}_{d}(\xi), \hat{\Psi}_{d}(\xi) \in \mathcal{L}_{2}$. In addition to time varying disturbances, constant, or slow varying, offsets will be introduced into the model. This is of particular interest when e.g. a constant negative mass flow disturbance pushes the equilibrium over the surge line, initiating surge or rotating stall. The offsets in mass flow and pressure rise is termed $d_{\phi}$ and $d_{\psi}$, respectively. The constant bias $d_{\psi}$ in pressure can also be thought of as reflecting some uncertainty in the compressor characteristic $\hat{\Psi}_{c}(\hat{\phi})$, and likewise the mass flow bias $d_{\phi}$ can be thought of as reflecting an uncertainty in the throttle characteristic $\hat{\Phi}(\hat{\psi})$. A study of surge/stall control for compressors with uncertain compressor characteristic can also be found in [15]. With these disturbances the model becomes:

$$
\begin{aligned}
\dot{\hat{\psi}} & =\frac{1}{4 B^{2} l_{c}}\left(\hat{\phi}-\hat{\Phi}_{T}(\hat{\psi})-d_{\phi}+\hat{\Phi}_{d}(\xi)\right) \\
\dot{\hat{\phi}} & =\frac{1}{l_{c}}\left(\hat{\Psi}_{c}(\hat{\phi})-\hat{\psi}-\hat{\Psi}_{d}(\xi)-d_{\psi}-u\right) .
\end{aligned}
$$

\section{Controller design}

Now, controllers are designed for the two separate cases of constant and time varying disturbances.

\subsection{Adaption of biases}

Adaptive backstepping [14] will now be employed to stabilize (8) with the time varying disturbances $\hat{\Phi}_{d}(\xi)$ and $\hat{\Psi}_{d}(\xi)$ set to zero. Two adaption laws will be designed in order to estimate the unknown constant biases and allow them to be counteracted by the control. 
Theorem 1 The controller

$u=\frac{l_{c}}{\gamma_{1}} z_{1}+c_{2} z_{2}-k_{3}\left(\bar{d}_{\phi}^{3}+3 \bar{d}_{\phi} z_{2}^{2}\right)-k_{2} \hat{\phi}^{2}-k_{1} \bar{d}_{\phi}+\bar{d}_{\psi}$,

where the estimates $\bar{d}_{\phi}$ and $\bar{d}_{\psi}$ are updated with

$$
\dot{\bar{d}}_{\phi}=-\frac{1}{\gamma_{1}} z_{1} \text { and } \dot{\bar{d}}_{\psi}=-\frac{1}{\gamma_{2}} z_{2},
$$

and $\frac{1}{\gamma_{1}}$ and $\frac{1}{\gamma_{2}}$ are adaption gains, makes the equilibrium of (8) globally asymptotically stable. The states of the model converges to their equilibrium values and the parameter error dynamics are globally asymptotically stable.

Proof: Adaptive backstepping is used

Step 1. The error variables $z_{1}$ and $z_{2}$ are defined as $z_{1}=\hat{\psi}$ and $z_{2}=\hat{\phi}-\alpha$. The first clf, $V_{1}$, is chosen as

$$
V_{1}=2 B^{2} l_{c} z_{1}^{2}+\frac{\gamma_{1}}{2} \tilde{d}_{\phi}^{2},
$$

where $\tilde{d}_{\phi} \triangleq d_{\phi}-\bar{d}_{\phi}$, is the parameter error and $\bar{d}_{\phi}$ is an estimate of $d_{\phi}$. The time derivative of $V_{1}$ now is

$$
\dot{V}_{1}=z_{1}\left(-\hat{\Phi}_{T}\left(z_{1}\right)+z_{2}-d_{\phi}+\alpha\right)-\gamma_{1} \tilde{d}_{\phi} \dot{\bar{d}}_{\phi},
$$

where (8) is used. Let the virtual control $\alpha$ be chosen as

$$
\alpha=\bar{d}_{\phi},
$$

and the estimate $\bar{d}_{\phi}$ be updated as

$$
\dot{\bar{d}}_{\phi}=-\frac{1}{\gamma_{1}} z_{1} \text {. }
$$

Thus, the terms including $\tilde{d}_{\phi}$ in (12) are cancelled out, and $\dot{V}_{1}$ can now be written as

$$
\dot{V}_{1}=z_{1} z_{2}-\hat{\Phi}_{T}\left(z_{1}\right) z_{1} \text {. }
$$

Step 2. The second clf is chosen as

$$
V_{2}=V_{1}+\frac{l_{c}}{2} z_{2}^{2}+\frac{\gamma_{2}}{2} \tilde{d}_{\psi}^{2}=\frac{1}{2} \boldsymbol{z}^{\mathrm{T}} \boldsymbol{P} \boldsymbol{z}+\frac{1}{2} \tilde{\boldsymbol{d}}^{\mathrm{T}} \boldsymbol{\Gamma}^{-1} \tilde{\boldsymbol{d}},
$$

where $\tilde{d}_{\psi} \triangleq d_{\psi}-\bar{d}_{\psi}$, is the parameter error, $\bar{d}_{\psi}$ is an estimate of $d_{\psi}, \tilde{\boldsymbol{d}}=\left(\begin{array}{ll}\tilde{d}_{\phi} & \tilde{d}_{\psi}\end{array}\right)^{\mathrm{T}}$, and $\boldsymbol{z}=\left(\begin{array}{ll}z_{1} & z_{2}\end{array}\right)^{\mathrm{T}}$. The matrices

$$
\boldsymbol{P}=\left(\begin{array}{cc}
4 B^{2} l_{c} & 0 \\
0 & l_{c}
\end{array}\right) \text { and } \boldsymbol{\Gamma}=\left(\begin{array}{cc}
\gamma_{1} & 0 \\
0 & \gamma_{2}
\end{array}\right)
$$

are constant and positive definite. Using (8), $\dot{V}_{2}$ is calculated as

$\dot{V}_{2}=-\hat{\Phi}_{T}\left(z_{1}\right) z_{1}+z_{2}\left(\hat{\Psi}_{c}(\hat{\phi})-u+\frac{l_{c}}{\gamma_{1}} z_{1}-d_{\psi}\right)-\gamma_{2} \tilde{d}_{\psi} \dot{\bar{d}}_{\psi}$.

Let the estimate $\bar{d}_{\psi}$ be updated as

$$
\dot{\bar{d}}_{\psi}=-\frac{1}{\gamma_{2}} z_{2}
$$

and the control be chosen as (9). By this choice of $u$, it is shown in [4] that $\dot{V}_{2}$ can be upper bounded as

$\dot{V}_{2} \leq-\left(c_{2}+k_{1}\right) z_{2}^{2}-k_{3}\left(z_{2}^{4}+3 \alpha^{2} z_{2}^{2}\right)-\hat{\Phi}_{T}\left(z_{1}\right) z_{1}$. (19) The reason for chosing $u$ in this way is to cancel out only the non-beneficial terms in the $z_{2} \hat{\psi}_{c}(\hat{\phi})$ part of $\dot{V}_{2}$, and leaving the stabilizing terms. By inserting the update laws (14) and (18) and the control (9) in (8), it can be shown that the error dynamics get the following form

$$
\begin{aligned}
\dot{\boldsymbol{z}} & =\boldsymbol{P}^{-1}(\boldsymbol{f}(\boldsymbol{z}, \tilde{\boldsymbol{d}})+\boldsymbol{S} \boldsymbol{z}+\tilde{\boldsymbol{d}}) \\
\dot{\tilde{d}} & =-\boldsymbol{\Gamma}^{-1} \boldsymbol{z},
\end{aligned}
$$

where

$$
\boldsymbol{f}(\boldsymbol{z}, \tilde{\boldsymbol{d}})=\left(\begin{array}{c}
-\hat{\Phi}_{T}\left(z_{1}\right) \\
-c_{2} z_{2}-k_{3}\left(z_{2}^{3}+3 \alpha^{2} z_{2}\right)
\end{array}\right)
$$

and

$$
\boldsymbol{S}=\left(\begin{array}{cc}
0 & 1 \\
-1 & 0
\end{array}\right)
$$

The stability result follows from application of LaSalle's theorem. From (19) it is seen that

$$
\dot{V}_{2} \equiv 0 \Rightarrow \boldsymbol{z} \equiv 0 \Rightarrow \dot{z} \equiv 0
$$

Inserting (24) into (20) we find that $\tilde{\boldsymbol{d}} \equiv 0$. Thus the origin of (20) is GAS.

Remark 1 The stability properties of the proposed scheme of two parameter update laws and control law, can also be established through passivity analysis of the error dynamics, [4]. As the mapping $\tilde{\boldsymbol{d}} \mapsto \boldsymbol{z}$ can be shown to be strictly passive, and the mapping $\boldsymbol{z} \mapsto-\tilde{\boldsymbol{d}}$ is a passive integrator, the feedback interconnection is globally stable, and $\boldsymbol{z}(\xi)$ converges to the origin.

Remark 2 The result of this section also holds if either of the two constant disturbances are set to zero. In the case of $d_{\phi} \equiv 0$ and $d_{\psi} \neq 0$, the controller (9) with parameter update law (10) degenerates to an ordinary linear PI-control law in $z_{2}$ :

$$
u=c_{2} z_{2}-\int_{0}^{t} \frac{1}{\gamma_{2}} z_{2}(\sigma) d \sigma .
$$

By the same arguments as in the above proof, $\boldsymbol{z}=0$ is GAS and $\bar{d}_{\psi}=\int_{0}^{t} \frac{1}{\gamma_{2}} z_{2}(\sigma) d \sigma$ converges to the true value of $d_{\psi}$.

\subsection{Rejection of time varying disturbances}

In [4], backstepping was employed to derive a stabilizing control law when time varying disturbances were taken into account. The control law resulting from this approach is given by

$$
\begin{aligned}
u= & c_{2} z_{2}-k_{3}\left(\alpha^{3}+3 \alpha z_{2}^{2}\right)-k_{2} \hat{\phi}^{2}-k_{1} \alpha \\
& +\frac{d_{1}}{4 B^{2}}\left(-\hat{\Phi}_{T}\left(z_{1}\right)+\hat{\phi}\right)+d_{2} z_{2}\left(1+\frac{d_{1}^{2}}{4 B^{2}}\right) .
\end{aligned}
$$

Here, passivity will be used to derive a stabilizing controller for (8) with all the disturbances set to zero. Then, $\mathcal{L}_{2}$-disturbances are included in the model and the stability result is shown to hold. The motivation for doing this is that the surge controller (26) resulting from the backstepping approach, when disturbances are included in the model, seems unnecessary complicated.

Passivity and input/output methods have been used in many control applications such as mechanical systems in general, electrical machines, marine vehicles and so on. Preliminary work on applying passivity to the compressor surge control problem was presented in [6].Of particular interest here will be, if the use of input/output methods to design controllers with disturbance rejection capabilities.

A brief introduction to passivity and $\mathcal{L}_{2}$ is given here. For a comprehensive treatment of these concepts, consult [20], from which the notation is taken. The signal space $\mathcal{L}_{2}$ consists, somewhat simplified, of all functions 
$f: \mathbb{R}_{+} \rightarrow \mathbb{R}$ that satisfy

$$
\int_{0}^{\infty}|f(\xi)|^{2} d \xi<\infty
$$

The truncation of $f$ to $[0, T]$ is defined as

$$
f_{T}(\xi)=\left\{\begin{array}{cc}
f(\xi) & , \quad 0 \leq \xi \leq T \\
0 & , \quad
\end{array},\right.
$$

and the set $\mathcal{L}_{2 e}$, the extension of $\mathcal{L}_{2}$, consists of all functions $f$ such that $f_{T} \in \mathcal{L}_{2}$. A mapping $\mathcal{G}: u \mapsto y$ with input $u \in \mathcal{L}_{2 e}$ and output $y \in \mathcal{L}_{2 e}$ is said to be passive if there exists a constant $\beta$ so that

$$
\int_{0}^{T} u(\xi) y(\xi) d \xi \geq \beta
$$

for all $u \in L_{2 e}$ and all $T \geq 0$. The inner product on $\mathcal{L}_{2 e}$ is

$$
\langle u, y\rangle_{T}=\int_{0}^{T} u(\xi) y(\xi) d \xi,
$$

and the truncated norm is

$$
\|u\|_{T}^{2}=\langle u, u\rangle_{T} .
$$

A concept that will be used in this chapter is that of strict output passivity. The mapping $\mathcal{G}: u \mapsto y$ is strict output passive if $\exists \kappa>0$ and $\exists \beta$ such that

$$
\langle y, u\rangle=\langle\mathcal{G} u, u\rangle \geq \kappa\|\mathcal{G} u\|_{T}^{2}+\beta \forall u \in \mathcal{L}_{2 e}, \forall T \geq 0 .
$$

The approach will be as follows: First, the basic model (1) without disturbances is studied, and certain passivity properties for this model are found. Then, disturbances are included in the analysis, and the results are generalized to this case. The following assumption will be needed in the passivity analysis:

Assumption 1 The throttle is assumed to be a passive component, moreover the constant $\kappa_{2}>0$ can always be chosen sufficiently small so that the characteristic satisfies the sector condition:

$$
\forall \hat{\psi} \exists \kappa_{2} \text { such that } \hat{\Phi}_{T}(\hat{\psi}) \hat{\psi} \geq \kappa_{2} \hat{\psi}^{2} .
$$

Our aim will be to design a control law $u=\hat{\Psi}_{v}(\hat{\phi})$ for the valve such that the compressor also can be operated stably on the left side of the original surge line without going into surge.

Passivity of flow dynamics: Consider the nonnegative function

$$
V_{1}(\hat{\phi})=\frac{l_{c}}{2} \hat{\phi}^{2}
$$

The time derivative of (34) along solution trajectories of $(5)$ is

$$
\dot{V}_{1}=-\hat{\psi} \hat{\phi}+\Psi_{e}(\hat{\phi}) \hat{\phi}
$$

Then, using (30) and (35) it follows that

$$
\begin{aligned}
\langle-\hat{\psi}, \hat{\phi}\rangle_{T} & =\left\langle-\hat{\Psi}_{e}(\hat{\phi}), \hat{\phi}\right\rangle_{T}+V_{1}(\xi)-V_{1}(0) \\
& \geq\left\langle-\hat{\Psi}_{e}(\hat{\phi}), \hat{\phi}\right\rangle_{T}-V_{1}(0)
\end{aligned}
$$

Hence, the flow dynamics

$$
\mathcal{G}_{1}:-\hat{\psi} \mapsto \hat{\phi}
$$

where $\mathcal{G}_{1}: \mathcal{L}_{2 e} \rightarrow \mathcal{L}_{2 e}$ is an input-output mapping, can be given certain passivity properties if the equivalent compressor characteristic $\hat{\Psi}_{e}(\hat{\phi})$ can be shaped appropriately by selecting the valve control law $\hat{\Psi}_{v}(\hat{\phi})$.

Passivity of pressure dynamics:
Proposition 1 The pressure dynamics

$$
\mathcal{G}_{2}: \hat{\phi} \mapsto \hat{\psi}
$$

where $\mathcal{G}_{2}: \mathcal{L}_{2 e} \rightarrow \mathcal{L}_{2 e}$ is an input-output mapping, are strictly output passive.

Proof :

Consider the nonnegative function

$$
V_{2}(\hat{\psi})=2 B^{2} l_{c} \hat{\psi}^{2} .
$$

Differentiating $V_{2}$ along the solution trajectories of (5) gives

$$
\dot{V}_{2}=\hat{\psi} \hat{\phi}-\hat{\phi}(\hat{\psi}) \hat{\psi} \text {. }
$$

In view of Assumption 1 and (40) it follows that

Hence,

$$
\begin{aligned}
\langle\hat{\psi}, \hat{\phi}\rangle_{T} & =\langle\hat{\phi}(\hat{\psi}), \hat{\psi}\rangle_{T}+\int_{0}^{T} \dot{V}_{2} d \xi \\
& \geq \kappa_{2} \int_{0}^{T} \psi^{2}(\xi) d \xi+V_{2}(\xi)-V_{2}(0) \\
& \geq \kappa_{2}\|\hat{\psi}\|_{T}^{2}-V_{2}(0)
\end{aligned}
$$

$$
\left\langle\mathcal{G}_{2} \hat{\phi}, \hat{\phi}\right\rangle_{T} \geq \kappa_{2}\left\|\mathcal{G}_{2} \hat{\phi}\right\|_{T}^{2}-V_{2}(0),
$$

and $\mathcal{G}_{2}$ is strictly output passive according to Definition 2.2 .1 in [20].

Proposition 2 Let the control law be given by

$$
\hat{\Psi}_{v}=c \hat{\phi}
$$

where $c>\frac{k_{2}^{2}}{4 k_{3}}-k_{1}+\kappa_{1}$ and $\kappa_{1}>0$ is a design parameter. Then the equivalent compressor characteristic $-\hat{\Psi}_{e}(\hat{\phi})$ will satisfy the sector condition

$$
\left\langle-\hat{\Psi}_{e}(\hat{\phi}), \hat{\phi}\right\rangle_{T} \geq \int_{0}^{T} \kappa_{1} \hat{\phi}^{2}(\xi) d \xi=\kappa_{1}\|\hat{\phi}\|_{T}^{2}
$$

\section{Proof :}

The compressor characteristic is defined in equation (6). The equivalent compressor characteristic $\hat{\Psi}_{e}(\hat{\phi})$ is then given by

$$
\hat{\Psi}_{e}(\hat{\phi})=-k_{3} \hat{\phi}^{3}(\xi)-k_{2} \hat{\phi}^{2}(\xi)-\left(k_{1}+c\right) \hat{\phi}(\xi)
$$

Consider the inner product

$$
\left\langle-\hat{\Psi}_{e}(\hat{\phi}), \hat{\phi}\right\rangle_{T}=\int_{0}^{T} \hat{\phi}^{2}(\xi) K(\hat{\phi}(\xi)) d \xi,
$$

where $K(\hat{\phi}(\xi)) \triangleq k_{3} \hat{\phi}^{2}(\xi)+k_{2} \hat{\phi}(\xi)+\left(k_{1}+c\right)$. It is noted that $K(\hat{\phi})$ has a minimum value for $\hat{\phi}=-\frac{k_{2}}{2 k_{3}}$. This minimum is calculated to be

$$
K(\hat{\phi})=k_{3} \hat{\phi}^{2}+k_{2} \hat{\phi}+\left(k_{1}+c\right) \geq-\frac{k_{2}^{2}}{4 k_{3}}+k_{1} .
$$

With the choice $c \geq \frac{k_{2}^{2}}{4 k_{3}}-k_{1}+\kappa_{1}$ it it follows from (45) that

$$
k_{3} \hat{\phi}^{2}(\xi)+k_{2} \hat{\phi}(\xi)+\left(k_{1}+c\right) \geq \kappa_{1} .
$$

By inserting (48) into (46), the result follows.

Provided $u=\hat{\Psi}_{v}$ is chosen as (43), it follows that also the flow dynamics are made strictly output passive, that is

$$
\left\langle\mathcal{G}_{1}(-\hat{\psi}),-\hat{\psi}\right\rangle_{T} \geq \kappa_{1}\left\|\mathcal{G}_{1}(-\hat{\psi})\right\|_{T}^{2}-V_{1}(0),
$$




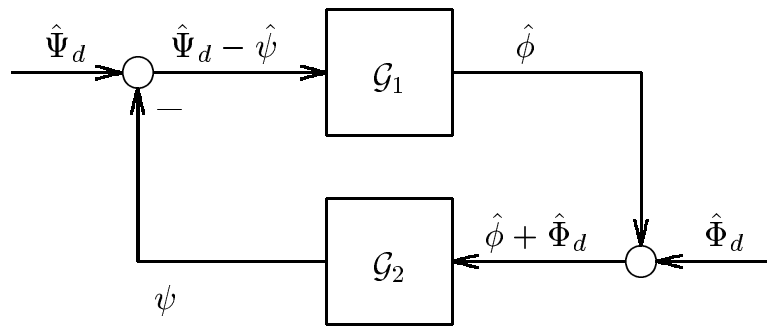

Figure 2: The closed loop system $\Sigma_{\mathcal{G}_{1}, \mathcal{G}_{2}}$ with disturbances

We now state a stability result for the closed loop system $\Sigma_{\mathcal{G}_{1}, \mathcal{G}_{2}}$ without disturbances. The closed loop system is shown in Figure 2 with disturbances.

Theorem 2 The closed loop system $\Sigma_{\mathcal{G}_{1}, \mathcal{G}_{2}}$ consisting of the model (5) and control law (43) is $\mathcal{L}_{2}$-stable. $\square$

\section{Proof :}

The closed loop system $\Sigma_{\mathcal{G}_{1}, \mathcal{G}_{2}}$ is a feedback interconnection of the two systems $\mathcal{G}_{1}:-\hat{\psi} \mapsto \hat{\phi}$ and $\mathcal{G}_{2}: \hat{\phi} \mapsto \hat{\psi}$. As stated in (42) and (49), the two mappings satisfy

$$
\begin{aligned}
\left\langle-\hat{\psi}, \mathcal{G}_{1}(-\hat{\psi})\right\rangle & \geq \kappa_{1}\left\|\mathcal{G}_{1}(-\hat{\psi})\right\|_{T}^{2}-V_{1}(0) \\
\left\langle\hat{\phi}, \mathcal{G}_{2} \hat{\phi}\right\rangle & \geq \kappa_{2}\left\|\mathcal{G}_{2} \hat{\phi}\right\|_{T}^{2}-V_{2}(0)
\end{aligned}
$$

for all $T \geq 0$ and all $\hat{\phi}, \hat{\psi} \in \mathcal{L}_{2 e}$. According to the passivity theorem, Theorem 2.2.6 in [20], $\Sigma_{\mathcal{G}_{1}, \mathcal{G}_{2}}$ is $\mathcal{L}_{2}$-stable.

Consider the case when the compression system is subject to disturbances $\hat{\Phi}_{d}(\xi)$ in mass flow and $\hat{\Psi}_{d}(\xi)$ in pressure rise. The model is repeated here for convenience:

$$
\begin{aligned}
\dot{\hat{\psi}} & =\frac{1}{4 B^{2} l_{c}}\left(\hat{\phi}+\hat{\Phi}_{d}(\xi)-\hat{\Phi}_{T}(\hat{\psi})\right) \\
\dot{\hat{\phi}} & =\frac{1}{l_{c}}\left(\hat{\Psi}_{c}(\hat{\phi})-u-\hat{\psi}-\hat{\Psi}_{d}(\xi)\right)
\end{aligned}
$$

It is assumed that $\hat{\Psi}_{d}(\xi), \hat{\Phi}_{d}(\xi) \in \mathcal{L}_{2 e}$. A stability result for the system shown in Figure 2 is now stated:

Theorem 3 The system (52) under control (43) is still $\mathcal{L}_{2}$-stable even if the disturbances $\hat{\Phi}_{d}(\xi)$ in mass flow and $\hat{\Psi}_{d}(\xi)$ in pressure rise are taken into account.

Proof :

Redefine $\mathcal{G}_{1}$ as $\mathcal{G}_{1}:-\left(\hat{\psi}-\hat{\psi}_{d}\right) \mapsto \hat{\phi}$ and $\mathcal{G}_{2}$ as $\mathcal{G}_{2}: \hat{\phi}+\hat{\phi}_{d} \mapsto \hat{\psi}$. The result follows by repeating the analysis in the preceding sections and replacing $-\hat{\psi}$ with $-\hat{\psi}+\hat{\psi}_{d}$ when establishing the strict output passivity of $\mathcal{G}_{1}$, and replacing $\hat{\phi}$ with $\hat{\phi}+\hat{\phi}_{d}$ when establishing the strict output passivity of $\mathcal{G}_{2}$.

The closed loop system is shown in Figure 2. Disturbance rejection of $\mathcal{L}_{2}$-disturbances in the Moore Greitzer model is also studied by [10], where throttle control of both surge and rotating stall is considered.

\section{Simulations}

Constant disturbances: A simulation of surge induced by a constant disturbance is showed in Figure 3. The compression system is initially operating stably with a throttle setting of $\gamma=0.65$ yielding a stable equilibrium. At $\xi=200$ the constant disturbances $d_{\phi}=-0.1$ and $d_{\psi}=0.05$ are introduced into the system, resulting in the state of the system being pushed over the surge line. Consequently, deep surge oscillations emerge. At $\xi=420$ the adaptive controller (9) with update laws (10) is switched on, and as can be seen the surge oscillations are brought to rest. The parameters of the controller were $c_{2}=1.1, \gamma_{1}=9$ and $\gamma_{2}=20$. The disturbances are unknown to the controller, but as guaranteed by Theorem 1 their estimates converges to the true values, which can be seen in the lower left plot of Figure 3. The pressure drop over the CCV is shown in the lower right plot of Figure 3.
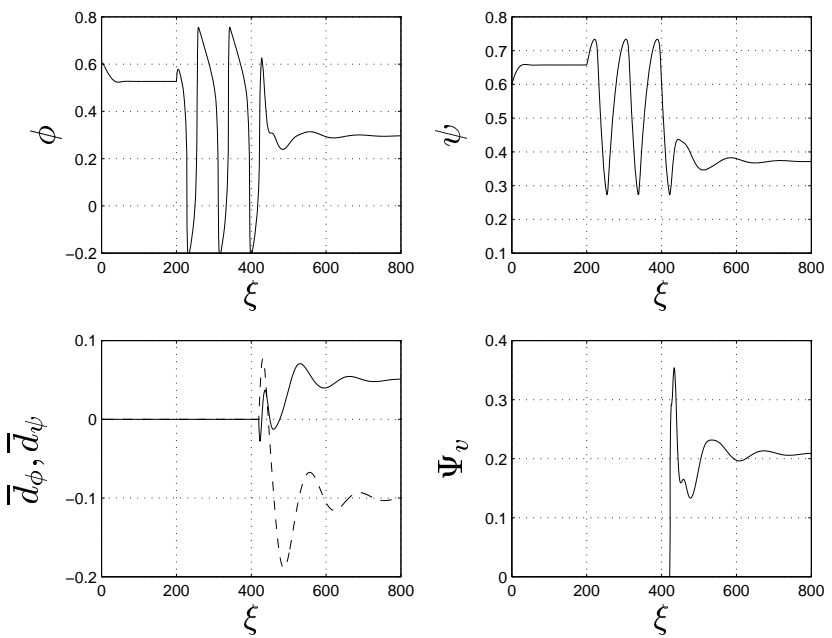

Figure 3: Disturbance induced surge stabilized by the adaptive controller (9).

Time varying disturbances: Now the system (8), with the constant biases set to zero, using control law (43) is simulated. The result is showed in Figure 4. The controller gain was set to $c=1.1$, and the controller was switched on at $\xi=300$. The disturbances were

$$
\begin{aligned}
& \hat{\Phi}_{d}(\xi)=0.25 e^{-0.0015 \xi} \cos (0.4 \xi) \\
& \hat{\Psi}_{d}(\xi)=0.2 e^{-0.0025 \xi} \sin (0.6 \xi)
\end{aligned}
$$

which has the same structure as the $\mathcal{L}_{2}$-disturbances considered in [10].

Also plotted (with dashed lines) in Figure 4 is the response of the controller (26), simulated with the same disturbances, and as can be seen, the two set of responses are almost indistinguishable. The parameters for (26) were chosen as $c_{2}=1, d_{1}=0.3, d_{2}=0.1$. The small difference in control action is due to the low damping $\left(d_{i}\right)$ chosen for $(26)$, but with the current disturbances that is all that was needed.

One advantage of the backstepping controller (26) compared to the passivity based (43) is that (26) ensures (shown in [4]) convergence to a set when the disturbances are not in $\mathcal{L}_{2}$, whereas the passivity approach used in the present paper, give no result in this case. 

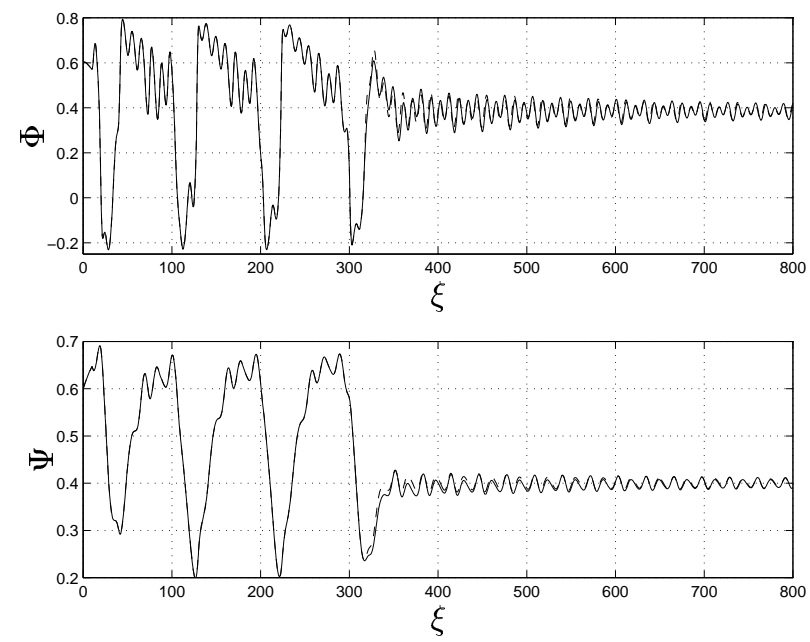

Figure 4: Comparison of closed loop response with passivity based and backstepping based controllers. The controllers were switched on at $\xi=400$.

\section{Concluding remarks}

By using adaptive backstepping, a nonlinear adaptive surge controller for a CCV in a compression system with constant disturbances in both mass flow and pressure rise was derived.

Using input/output theory and passivity it was possible to show that the a proportional controller for the CCV yields a $\mathcal{L}_{2}$-stable system in the presence of both time varying mass flow disturbances as well as time varying pressure disturbances.

It is straightforward to show that the stability result is still valid if the control law (43) is changed as long as the sector condition (44) hold. Thus, a more sophisticated controller could be used in order to e.g. minimize the steady state pressure drop over the CCV or improve transient performance, and still stability could be shown.

The passivity based method used here shows promise for developing a simple, low order, partial state feedback controller when rotating stall is also taken into account, and still achieving disturbance rejection. This is a subject under investigation.

\section{References}

[1] J.C. DeLaat, R.D. Southwick, and G.W. Gallops. High stability engine control (HISTEC). In Proceedings of $A I A A / S A E / A S M E / A S E E$ 32nd Joint Propulsion Conference, Lake Buena Vista, FL, July 1996. NASA TM 107272, AIAA-96-2586.

[2] J.L. Dussourd, G.W. Pfannebecker, and S.K. Singhania. An experimental investigation of the control of surge in radial compressors using close coupled resistances. Journal of Fluids Engineering, 99:64-76, 1977.

[3] J.B. Erskine and N. Hensman. Vibration induced by pump instability and surging. In Vibrations and noise in pump, fan, and compressor installations, pages 87-98, Southamton, UK, September 1975. The Institution of Mechanical Engineers, Mechanical Engineering Publications limited.
[4] J.T. Gravdahl. Surge and Rotating Stall in Compressors: Modeling and Control. Advances in Industrial Control. Springer-Verlag, London, 1998. Accepted for publication, to appear.

[5] J.T. Gravdahl and O. Egeland. Compressor surge control using a close-coupled valve and backstepping. In Proceedings of the $199^{7}$ American Control Conference, Albuquerque, NM, June 1997.

[6] J.T. Gravdahl and O. Egeland. Passivity based compressor surge control using a close-coupled valve. In A. Isidori and F. Allgöwer, editors, Proceedings of the 1997 COSY Workshop on Control of Nonlinear and Uncertain Systems, pages 139-143, Zurich, Switzerland, January 1997.

[7] E.M. Greitzer. Surge and Rotating stall in axial flow compressors, Part I: Theoretical compression system model. Journal of Engineering for Power, 98:190198, 1976.

[8] E.M. Greitzer. The stability of pumping systems - The 1980 Freeman scholar lecture. Journal of Fluids Engineering, 103:193-242, 1981.

[9] E.M. Greitzer and F.K. Moore. A theory of poststall transients in a axial compressor systems: Part IIApplication. Journal of Engineering for Gas Turbines and Power, 108:231-239, 1986.

[10] W.M. Haddad, J.L. Fausz, V.-S. Chellaboina, and A. Leonessa. Nonlinear robust disturbance rejection controllers for rotating stall and surge in axial flow compressors. In Proceedings of the 1997 International Conference on Control Applications, pages 767-772, Hartford, CT, October 1997.

[11] K.E. Hansen, P. Jørgensen, and P.S. Larsen. Experimental and theoretical study of surge in a small centrifugal compressor. Journal of Fluids Engineering, 103:391-394, 1981.

[12] T.P. Hynes and E.M. Greitzer. A method for assessing effects of circumferential flow distortion on compressor stability. Journal of Turbomachinery, 109:371379, 1987.

[13] W.M. Jungowski, M.H. Weiss, and G.R. Price. Pressure oscillations occurring in a centrifugal compressor system with and without passive and active surge control. Journal of Turbomachinery, 118:29-40, 1996.

[14] M. Krstić, I. Kanellakopoulos, and P.V. Kokotović. Nonlinear and Adaptive Control Design. John Wiley \& Sons Inc., 1995.

[15] A. Leonessa, V.-S. Chellaboina, and W.M. Haddad. Robust stabilization of axial flow compressors with uncertain pressure-flow maps. In Proceedings of the 1997 International Conference on Control Applications, pages 671-676, Hartford, CT, October 1997.

[16] J.S. Simon and L. Valavani. A Lyapunov based nonlinear control scheme for stabilizing a basic compression system using a close-coupled control valve. In Proceedings of the 1991 American Control Conference, pages 2398-2406, 1991.

[17] S. Simon. Feedback stabilization of compression systems. PhD thesis, MIT, February 1993.

[18] M. van de Wal and F. Willems. Selection of actuators and sensors for compressor control. Technical Report WFW 96.155, Eindhoven University of Technology, November 1996.

[19] M. van de Wal, F. Willems, and B. de Jager. Selection of actuators and sensors for active surge control. In Proceedings of the 1997 International Conference on Control Applications, pages 121-127, Hartford, CT, October 1997.

[20] A.J. van der Schaft. $L_{2}$-gain and passivity techniques in nonlinear control, volume 218 of Lecture notes in Control and Information Sciences. Springer-Verlag, Heidelberg, 1996. 